\title{
An uncommon cause of finger drop in a patient with rheumatoid arthritis
}

\author{
Stephen P McDonald, Malcolm D Smith
}

\section{Case report}

A 72 year old man with a four year history of seropositive rheumatoid arthritis had been treated with intramuscular and oral gold and methotrexate in the past, all of which were stopped because of toxicity. Despite previous poor disease control with hydroxychloroquine he had declined other disease modifying agents. There was no other significant medical history.

He presented with sudden onset of left shoulder and arm pain while reaching up with the left upper arm. Hours later he noted mild swelling and paraesthesiae in his left hand, and difficulty using the fingers of the hand. Examination of his shoulder showed anterosuperior subluxation of the humeral head, with severe restriction of all active and passive movements. There was an active left elbow synovitis with a moderate joint effusion. There was no weakness of the biceps or triceps muscles. Supination appeared to be slightly weak, but was difficult to assess initially due to elbow pain. Finger and thumb extension was absent but thumb abduction was intact. Wrist extension was weak, with radial deviation. Sensation was intact to all modes in the left upper limb.

Initial diagnoses attempted to link the shoulder and hand signs, and included a possible acute rotator cuff rupture with brachial plexus injury or reflex sympathetic dystrophy. An urgent shoulder arthrogram showed no evidence of rotator cuff tear, and there was immediate improvement in shoulder mobility after intra-articular depot steroids and local anaesthetic.

There was, however, no improvement in the left forearm or hand symptoms or signs, and it became clear there were two disease sites involved. Other processes, in particular extensor tendon rupture or a neuropraxia of the posterior interosseous nerve, were sought. Because of the difficulty eliciting clinical signs in an acutely inflamed forearm, opinions were sought from a neurologist and plastic surgeon, who both felt the clinical picture was typical of a posterior interosseous nerve lesion.

Nerve conduction studies showed no abnormality of radial, median, or ulnar nerve function. An arthrogram of the left elbow (figs 1and 2) showed marked irregularity suggesting an active synovitis, a large joint effusion and erosive bony changes. A computerised tomographic (CT) arthrogram showed no cystic extension of the joint capsule.

Steroids were injected into the left radiohumeral joint $(5.7 \mathrm{mg}$ Celestone Chronodose), and a dynamic hand splint was provided. One week later he was started on sulphasalazine. Finger extension returned slowly over the succeeding six months, and the elbow synovitis responded well to the combination of local steroids and sulphasalazine.

\section{Discussion}

Posterior interosseous nerve (PIN) lesions are only rarely seen in rheumatoid arthritis. Other causes of a PIN lesion include acute and chronic trauma, local tumours, and bicipital bursal enlargement from the nearby elbow joint, as well as a primary vasculitis or mononeuritis. ${ }^{12}$ The anatomical cause is compression of the nerve against the fibrous arcade of Frohse by a hypertrophied elbow joint capsule. The arcade of Frohse is a band formed by the proximal insertion of supinator lying immediately superficial to the anterior aspect of the elbow joint. In $30 \%$ of adults this is fibrous, which predisposes to a compressive PIN lesion. ${ }^{3}$

The posterior interosseous nerve arises from the radial nerve at the level of the radial head, then passes under a number of fibrous bands (the arcade of Frohse, at the radial head, distal border of supinator and beneath vascular bundles). The nerve is vulnerable to compression at all these points. It supplies motor fibres to supinator, finger and thumb extensors, abductor pollicis longus, and extensor carpi ulnaris, and sensory innervation to the intercarpal joints. ${ }^{3}$

The first case of PIN entrapment associated with rheumatoid arthritis was described in $1967,{ }^{4}$ and since then 10 further cases have been described in six more reports. ${ }^{5-10}$ In all cases the patients had long standing rheumatoid arthritis, and in all but one, local interventions-either intra-articular injection or surgery-were performed. At operation there was usually a bulging hypertrophied elbow synovium. A fibrous arcade of Frohse was seen in all but one case. A further case of 


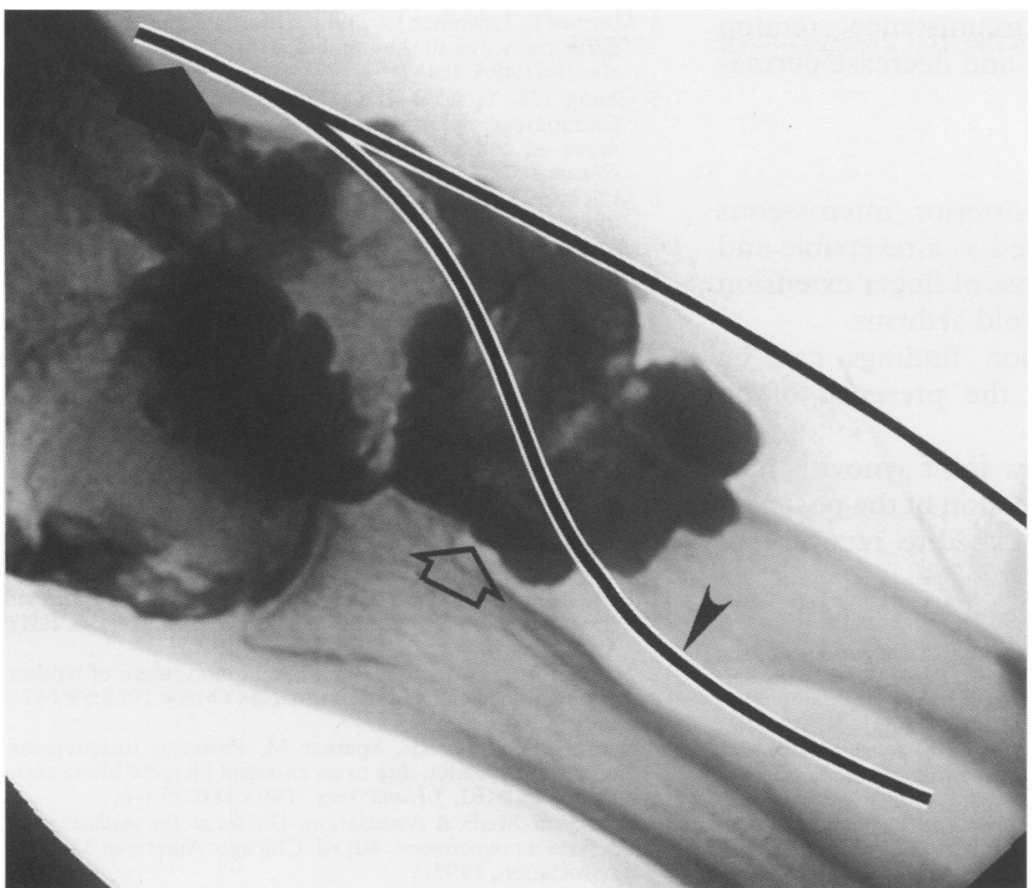

Figure 1 Arthrogram (left oblique view) of left elbow joint showing synovial hypertrophy (open arrowhead). The course of the radial nerve (large filled arrowhead) and posterior interosseous nerve (small filled arrowhead) have been marked on the $x$ ray.

radial nerve entrapment following synovial rupture of a rheumatoid elbow settled with both intra-articular and systemic steroids. ${ }^{11}$

Although in this case imaging did not directly demonstrate the posterior interosseous nerve, the diagnosis is a clinical one, and the anatomical location of the nerve strongly suggests compression and stretching by a hypertrophied and inflamed joint capsule as the cause. Resolution of the problem following intra-articular steroids and initiation of systemic treatment for rheumatoid arthritis was delayed, but in keeping with resolution of the elbow synovitis.

The primary differential diagnoses of a posterior interosseous nerve lesion are extensor tendon rupture or dislocation, and metacarpophalangeal joint dislocation. ${ }^{4} 57{ }^{10}$ Physical

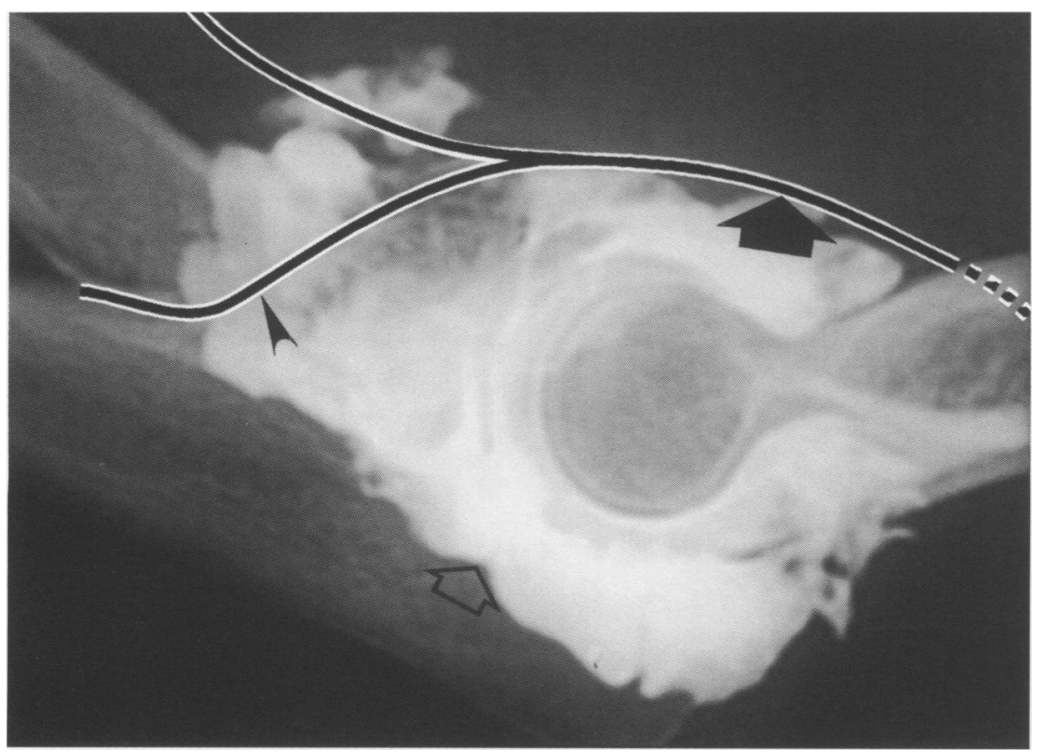

Figure 2 Arthrogram (lateral view) of left elbow joint showing synovial hypertrophy (open arrowhead). The course of the radial nerve (large filled arrowhead) and posterior interosseous nerve (small filled arrowhead) have been marked on the $x$ ray. signs can be difficult to elicit in an acutely inflamed forearm, but PIN lesions can be distinguished by involvement of thumb extension, whereas extensor tendon rupture usually affects the fourth and fifth digits and almost never the thumb. ${ }^{12}$ Tendon integrity is assessed by tenodesis (passive finger extension secondary to wrist flexion). Rupture usually occurs suddenly following chronic dorsal synovitis, and is associated with wrist pain. PIN lesions are said to be preceded by a period of local elbow pain, and are insidious in onset, ${ }^{10}$ although this case shows that this is not a reliable distinction. Finally, dislocation of the radial head can cause PIN compression but should be readily apparent on physical examination.

Tendon sheath radiography has been attempted to show tendinous integrity, ${ }^{5}$ with only limited success. The only definitive demonstration of rupture is at surgery, and in the first PIN lesion described ${ }^{4}$ tendon exploration was carried out before the diagnosis was recognised. For confirmation of PIN involvement electromyography can clarify the site and degree of nerve damage.

Elbow arthrography was used in this case and two other reports ${ }^{8}{ }^{9}$ to demonstrate the degree of synovial proliferation. In addition we performed CT arthrography to define the hypertrophied synovium more accurately. Although direct compression was not seen, the extension of contrast into areas adjacent to the PIN suggests a local effect via stretching of the nerve. The optimal modality to image the region of the PIN in the cubital fossa is magnetic resonance imaging (MRI). Although used in non-rheumatoid cases of compression by a bicipital bursa ${ }^{13}$ there are no reports of MRI scanning in rheumatoid arthritis cases.

Treatment options available are surgical exploration of the nerve, intra-articular steroid into the elbow, and systemic anti-inflammatory treatment. Most reports of this condition in rheumatoid arthritis have described surgery as the principal treatment, several investigators describing variable success with intra-articular steroids. In seven cases, either oral ${ }^{5}$ or intraarticular ${ }^{4-9}$ steroids were used. Of these, four proceeded to surgery, in one case because of a ruptured synovial cyst. Early use of intra-articular steroids appears to favour a more successful outcome, particularly if they are used within one week of onset of symptoms (for example, case 2 in ${ }^{6}$, case 3 in ${ }^{9}$ ), as was performed in this case.

If PIN lesions are found in rheumatoid arthritis, we suggest a trial of intra-articular steroid if the diagnosis is made early. Elbow synovitis is an important contributor to the pathogenesis of the neuropathy, and attempts should be made to control this either locally or systemically. Decompressive surgery should be contemplated if medical management fails, if there was a prolonged course before presentation, or if compression relates to synovial rupture. If function does not return, considerable disability usually results from loss of wrist and finger dorsiflexion, and has been considered to be equivalent to loss of $35 \%$ of the function of 
that limb. ${ }^{14}$ In these circumstance, tendon transfer may offer benefit and decrease permanent disability.

\section{The lesson}

- Compression of the posterior interosseous nerve should be considered as a reversible and treatable cause of acute loss of finger extension in a patient with rheumatoid arthritis.

- History and examination findings can be difficult to interpret in the presence of an acutely inflamed joint.

- Local treatment of elbow joint synovitis may assist in removing compression of the posterior interosseous nerve and expedite recovery of nerve function.

1 Mulholland RC. Non-traumatic progressive paralysis of the posterior interosseous nerve. $\mathcal{F}$ Bone foint Surg $\mathrm{Br}$ posterior interossec

2 Hazihume H, Inoue H, Nagashima K, Hamaya K. Posterior interosseous nerve paralysis related to focal radial nerve constriction secondary to vasculitis. $\mathcal{f}$ Hand Surg 1993;18B:757-60.

3 Spinner $M$. The arcade of Frohse and its relationship to posterior interosseous nerve paralysis. F Bone foint Surg $\mathrm{Br}$ 1968;50B:809-12.
4 Marmor L, Lawrence JF, Dubois EL. Posterior interosseous nerve palsy due to rheumatoid arthritis. $\mathcal{F}$ Bone foint Surg Am 1967;49A:381-2.

5 Chang LW, Gowans JDC, Granger CV, Millender LH Entrapment neuropathy of the posterior interosseous nerve- a complication of rheumatoid arthritis. Arthritis Rheum 1972;15:350-2

6 Millender LH, Nalebuff EA, Holdsworth DE. Posterio interosseous nerve syndrome secondary to rheumatoid interosseous nerve syndrome secondary to the
synovitis. $₹$ Bone foint Surg Am 1973;55A:753-7.

7 Roth AI, Stulberg BN, Fleegler EJ, Belhobek GH. Elbow arthrography in the evaluation of posterior interosseous nerve compression in rheumatoid arthritis. $f$ Hand Surg 1985;11B:120-2.

8 White SH, Goodfellow JW, Mowat A. Posterior interosseous nerve palsy in rheumatoid arthritis. $f$ Bone foint Surg $\mathrm{Br}$ 1988;70B:468-71.

9 Ishikawa $\mathrm{H}$, Hirohata $\mathrm{K}$. Posterior interosseous nerve Ishikawa $\mathrm{H}$, Hirohata $\mathrm{K}$. Posterior interosseous nerve syndrome associated with rheumatoid synovial cy
elbow joint. Clin Orthop Rel Res 1990;254:134-9.

10 Fernandez AM, Tiku ML. Posterior interosseous nerve entrapment in rheumatoid arthritis. Semin Arthritis Rheum 1994;24:57-60.

11 Fernandes L, Goodwill CJ, Srivatsa SR. Synovial rupture of heumatoid elbow causing radial nerve compression. $B M$ 1979;i:17-18

12 Nalebuff EA, Potter TA. Rheumatoid inolvement of tendon and tendon sheaths in the hand. Clin Orthop 1968;59:14759.

13 Spinner RJ, Lins RE, Spinner M. Posterior interosseous nerve compression due to an enlarged bicipital bursa confirmed by MRI. F Hand Surg ? 1993;18B:753-6.

14 American Medical Association. Guides to the evaluation of permanent impairment, 4th ed. Chicago: American Medical Association, 1993. 